\title{
Photon-electron coincidence experiments at synchrotron radiation facilities with arbitrary bunch modes
}

Cite as: Rev. Sci. Instrum. 92, 045110 (2021); https://doi.org/10.1063/5.0040179

Submitted: 10 December 2020 • Accepted: 05 April 2021 • Published Online: 21 April 2021

C. Ozga, C. Honisch, Ph. Schmidt, et al.
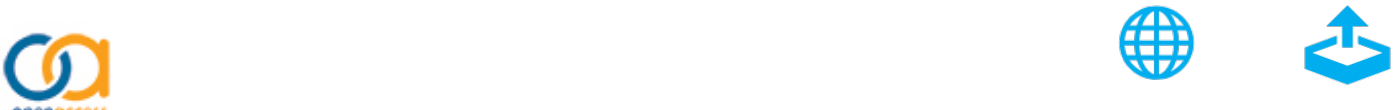

\section{ARTICLES YOU MAY BE INTERESTED IN}

Setup for multicoincidence experiments of photons in the extreme ultraviolet to visible spectral range and charged particles-The solid angle maximization approach Review of Scientific Instruments 90, 093104 (2019); https://doi.org/10.1063/1.5109104

Time- and momentum-resolved photoemission studies using time-of-flight momentum microscopy at a free-electron laser

Review of Scientific Instruments 91, 013109 (2020); https://doi.org/10.1063/1.5118777

Core level photoelectron spectroscopy of heterogeneous reactions at liquid-vapor interfaces: Current status, challenges, and prospects

The Journal of Chemical Physics 154, 060901 (2021); https://doi.org/10.1063/5.0036178

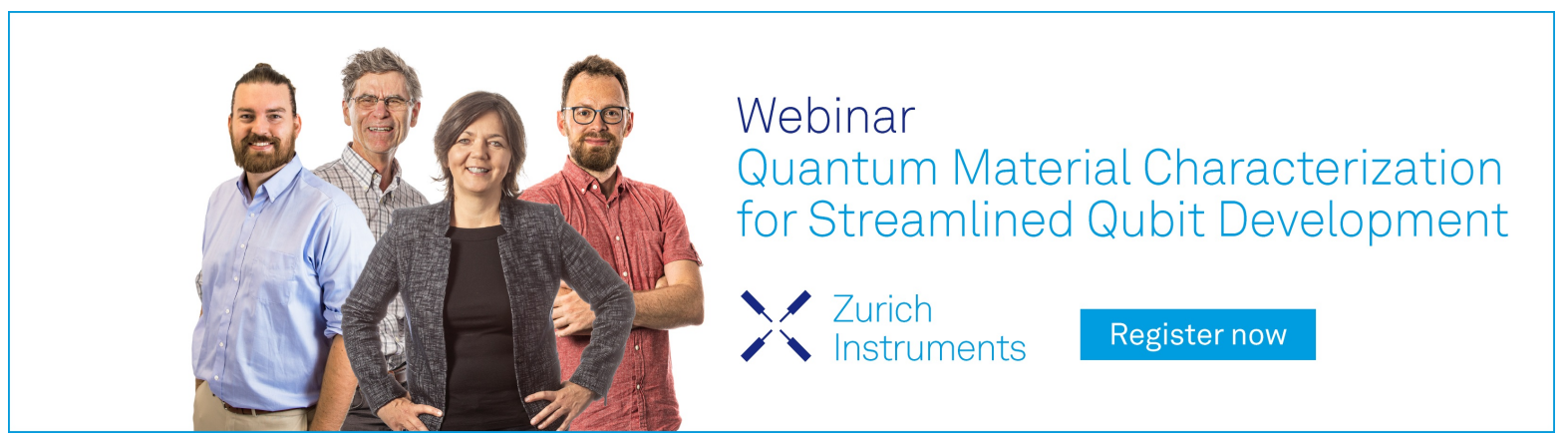




\title{
Photon-electron coincidence experiments at synchrotron radiation facilities with arbitrary bunch modes
}

\author{
Cite as: Rev. Sci. Instrum. 92, 045110 (2021); doi: 10.1063/5.0040179 \\ Submitted: 10 December 2020 - Accepted: 5 April 2021 • \\ Published Online: 21 April 2021
}

\begin{abstract}
C. Ozga,
C. Honisch, ${ }^{1}$ Ph. Schmidt,

X. Holzapfel,

C. Zindel, ' C. Küstner-Wetekam,

U. Hergenhahn,

A. Ehresmann,'

A. Knie, ' (D) and A. Hans'

AFFILIATIONS

${ }^{1}$ Institut für Physik und CINSaT, Universität Kassel, Heinrich-Plett-Straße 40, 34132 Kassel, Germany

${ }^{2}$ European XFEL GmbH, Holzkoppel 4, 22869 Schenefeld, Germany

${ }^{3}$ Leibniz-Institut für Oberflächenmodifizierung (IOM), Permoserstraße 15, 04318 Leipzig, Germany

${ }^{4}$ Fritz-Haber-Institut der Max-Planck-Gesellschaft, Faradayweg 4-6, 14195 Berlin, Germany
\end{abstract}

C. Richter,

a) Author to whom correspondence should be addressed: hans@physik.uni-kassel.de

\begin{abstract}
We report the adaptation of an electron-photon coincidence detection scheme to the multibunch hybrid mode of the synchrotron radiation source BESSY II (Helmholtz-Zentrum Berlin). Single-event-based data acquisition and evaluation, combined with the use of relative detection times between the coincident particles, enable the acquisition of proper coincidence signals from a quasi-continuous excitation pattern. The background signal produced by accidental coincidences in the time difference representation is modeled using the non-coincident electron and photon spectra. We validate the method by reproducing previously published results, which were obtained in the single bunch mode, and illustrate its usability for the multibunch hybrid mode by investigating the photoionization of $\mathrm{CO}_{2}$ into $\mathrm{CO}_{2}^{+} \mathrm{B}$ satellite states, followed by subsequent photon emission. The radiative lifetime obtained and the electron binding energy are in good agreement with earlier publications. We expect this method to be a useful tool to extend the versatility of coincident particle detection to arbitrary operation modes of synchrotron radiation facilities and other excitation sources without the need for additional experimental adjustments.
\end{abstract}

(C) 2021 Author(s). All article content, except where otherwise noted, is licensed under a Creative Commons Attribution (CC BY) license (http://creativecommons.org/licenses/by/4.0/). https://doi.org/10.1063/5.0040179

\section{INTRODUCTION}

Since its first use in the $1920 \mathrm{~s},{ }^{1,2}$ coincident particle detection evolved to one of the most powerful tools in various disciplines, e.g., nuclear science, ${ }^{3-7}$ high-energy particle physics, ${ }^{6,8-10}$ positronemission tomography, ${ }^{11,12}$ astrophysics, ${ }^{13,14}$ quantum communication, ${ }^{15,16}$ or atomic and molecular physics, ${ }^{17-27}$ and references therein. The simultaneous detection of multiple particles from decays of radioactive matter or following defined target-projectile interactions allowed the identification and quantification of distinct processes in experiments. If several processes occur simultaneously, the process of interest can be identified by specific correlations between the detected particles and their properties. Geometric and energetic reconstruction of fragmenting systems and the determination of their absolute configuration are thus possible. ${ }^{23,25}$ However, a complete presentation of the various adaptions is beyond the scope of this publication. Therefore, we limit ourselves to a few aspects, which we think are important for the work at hand.

Due to experimental limitations, for instance, finite relative detector quantum efficiencies $<1$, noise signals, or the accidental occurrence of two independent interactions, every coincident particle detection experiment needs to deal with accidental coincidences, i.e., uncorrelated signals that are mistakenly detected as coincident events. These are also called random, ${ }^{28}$ false, ${ }^{8,17,29}$ or chance ${ }^{7,30}$ coincidences. Therefore, coincidence experiments rely on the treatment of these accidental coincidences using a wide variety of methods, where the actual used method depends on the experimental setup, data acquisition, and the investigated physical processes. Some examples of these methods are the usage of multiple detection windows ${ }^{31-34}$ and modeling of the underlying processes ${ }^{35-40}$ 
as well as state-of-the-art artificial neural networks, ${ }^{10}$ and they are constantly evolving.

In the particular field of atomic and molecular physics, coincident particle detection upon electronic excitation of atoms or molecules often relies on measurements, in which the times of detection of individual reaction products relative to a joint reference time are recorded. The time differences between the coincident particles are then the basis to conjecture on the correlations of the detected particles at the time of their emergence. The reference time usually has an arbitrary but constant offset to the start of the process of interest and needs to be synchronized with it. Experimentally synchronized reference times may be realized by using either pulsed target delivery or, for interaction-type experiments, pulsed projectile beams with sufficiently short pulse widths. For an unequivocal correlation between the measurement time and the reference time, the repetition rate in both types of experiments must be sufficiently low to allow the particles to traverse through an optionally used spectrometer and to reach the detector between two consecutive pulses. If the measurement times exceed the temporal spacing between two exciting pulses, their relative difference should at least be shorter. Since pulsed target delivery with these properties is highly challenging, in most of the experimental settings, pulsed excitation is used. A further boundary condition for successful interaction-type coincidence experiments is imposed by the number of simultaneously occurring processes from different interacting particles due to high particle densities. This number needs to be small enough to ensure the unambiguous connection between a process started by the interaction with some particle and the particles recorded as a result of that process. This condition can be met by tuning either the target or the projectile density. Alternatively, a reliable procedure for removal of random coincidences is required, based on statistical considerations, as, e.g., in the present work.

A more general approach to coincident particle detection uses the detection of one particle as a time reference, and the detection times of the other particles are measured relative to this reference. ${ }^{19,41-51}$ Here, no joint and synchronized reference clock is required, which allows the use of continuous or quasi-continuous excitation sources additionally to pulsed sources.

Synchrotron radiation, which is inherently pulsed due to its generation by electron bunches circulating in a storage ring, is well suited for photoionization and photoexcitation coincidence experiments. The temporal bunch filling pattern (bunch mode) of the ring determines the time distance between successive synchrotron radiation flashes initializing photon-interaction driven processes. For an adequate temporal spacing between consecutive excitation pulses, the synchrotron radiation facilities are optionally operated using single bunch or few bunch filling patterns with only one or few electron bunches circulating in the storage ring. Since these operation modes imply comparably low photon flux for other experimental techniques, they are often provided for a strictly limited time per year. Consequently, other experimental techniques have been developed to perform coincidence experiments with other bunch filling patterns. For example, different electron orbits for different fill patterns, ${ }^{52}$ pulse picking by resonant excitation, ${ }^{53,54}$ or mechanical and electrical chopping to access the single bunch window of a hybrid fill pattern ${ }^{55-57}$ have been used. Yet, these techniques rely on either advanced capabilities of the synchrotron facility itself or synchronization of the fill pattern with an additional chopper. In any case, only a small portion of the available photons can be used by these techniques, which often drastically increases the required acquisition times.

Without additional preparation of the exciting photon beam, the use of pulsed excitation sources in interaction-type experiments with a temporal spacing between excitation pulses smaller than the traversal times of the particles through the corresponding spectrometers may introduce a structured background of accidental coincidences, complicating its removal considerably. This has been discussed extensively for solid-state Auger electron-photoelectron coincidence measurements using electrostatic electron analyzers, ${ }^{58,59}$ including a model for an estimate of the contribution of accidental coincidences to the total coincidence count rate. This method has later been adapted to gas-phase photo-double-ionization experiments also using electrostatic electron analyzers. ${ }^{60}$ Additionally, it was extended to Auger electron-photoelectron coincidence measurements combining an electrostatic electron analyzer with an electron time-of-flight detector ${ }^{61}$ using the multibunch hybrid mode of the third generation synchrotron radiation facility BESSY II. ${ }^{56,62,63}$ Here, the time signal of the electrostatic electron analyzer serves as a reference signal to the nearly fixed time-of-flight of the passing electrons ${ }^{61}$ except for a tunable time spread. ${ }^{61,64,65}$ Independently, a similar approach for the removal of the accidental background was proposed for coincident particle detection of neutron induced fission experiments using waveform digitizers ${ }^{7}$ based on digital coincidence counting methods. ${ }^{66-68}$ For a minimum temporal distance of two subsequent excitation pulses of $1800 \mathrm{~ns}$, how a software based sorting and post-processing of the detected signals can be used to obtain the estimate of the accidental background was shown.

In this work, we use our recently developed experimental setup for photon-electron coincidence experiments ${ }^{69}$ without any additional preparation of the setup or the incident photon beam for measurements in the multibunch hybrid mode of BESSY II. We show that a background subtraction routine similar to the one developed for neutron induced fission experiments ${ }^{7}$ for well separated excitation pulses can also be applied to electron-photon coincidence measurements for a minimal temporal distance of the exciting pulses of $2 \mathrm{~ns}$ and effectively eliminates the background of accidental coincidences. Therefore, the approach used for Auger electron-photoelectron coincidences ${ }^{59,61}$ can be extended to coincident particle detection schemes involving the detection time measurements of the emitted photons. As a result, the restrictions given by the provided fill patterns of the excitation sources can be lifted for any coincidence experiment where the emitted photons need to be measured. The application of this evaluation scheme to the data acquired previously in the single bunch mode shows the flexibility of this method based on singleevent acquisition using state-of-the-art time-to-digital converters (TDCs)

This publication is arranged as follows: The general experiment and data acquisition are described in Sec. II. Next, we revisit the accidental coincidence background subtraction method for event-based coincidence detection in Sec. III. The performance of the discussed method is shown in Sec. IV for $2 p$ photoionization of Ar clusters in the single bunch operation mode and outer valence photoionization of $\mathrm{CO}_{2}$ in the multibunch hybrid mode. We summarize in Sec. V. 


\section{EXPERIMENT AND DATA ACQUISITION}

The experiment on $\mathrm{CO}_{2}$ photoionization was conducted at the U125/2-10 m NIM beamline of the BESSY II storage ring of the Helmholtz-Zentrum Berlin (HZB). ${ }^{70,71}$ The synchrotron was operated in the Multi-Bunch Hybrid Top-Up (MBH) mode. At BESSY II, 400 phase space buckets can be filled with electron bunches. With $800 \mathrm{~ns}$ circulation time, this corresponds to an average time difference of 2 ns between the buckets. In the MBH mode, 300 buckets (out of 400 buckets) are filled with electron bunches ${ }^{56,63}$ with a dark gap of 100 buckets as shown in Fig. 1. Additionally, a single camshaft pulse is inserted in the center of the dark gap ${ }^{72}$ and a PPRE pulse (Pulse Picking by Resonant Excitation) is inserted three buckets before the multibunch part of the fill pattern, ${ }^{54,72}$ i.e., electron bunches are stored in 302 of 400 available buckets.

The targets were produced by supersonic expansion of gaseous samples through a conical copper nozzle $\left(80 \mu \mathrm{m}\right.$ orifice, $15^{\circ}$ half opening angle). Additional measurements were performed using heterogeneous $\mathrm{NeKr}$ clusters for a time-of-flight to kinetic energy conversion of the $\mathrm{CO}_{2}$ measurement. For this purpose, Ne gas with an admixture of $1.2 \%-2.0 \% \mathrm{Kr}$ at a stagnation pressure of $p=1.1 \mathrm{bar}$ was expanded through the nozzle, which was cooled to $T=90 \mathrm{~K}$ using a liquid nitrogen cryostat (for details, see Ref. 73). This target was chosen since detailed information on photon-electron coincidence spectra was available obtained with the same setup. ${ }^{73}$ Measurements on gaseous $\mathrm{CO}_{2}$ were performed using the same source at $T=243 \mathrm{~K}$ and $p=0.49$ bar to reduce the signal contribution of photoionization processes stemming from vibrationally excited neutral $\mathrm{CO}_{2} \cdot{ }^{74}$

The data evaluation procedure presented in this publication has also been applied to datasets obtained in a previous experiment in the single bunch operation mode. Target preparation and the experimental setup were similar to the experiment presented here and have been described in detail elsewhere. ${ }^{69,73}$ For both experiments, electrons were detected by a short magnetic-bottle-type time-of-flight electron spectrometer with a drift tube length of $600 \mathrm{~mm}^{75}$ and the emitted photons were detected with a single-photon counting detector. ${ }^{69,76,77}$ The photon detector was equipped with a $\mathrm{MgF}_{2}$ window and a CsTe photocathode enabling the detection of photons in the wavelength range of $120-300 \mathrm{~nm}$ (about 10.4-4.0 eV). ${ }^{69}$ For optimum coincidence efficiency in both experiments, the solid angle of the electron detection has been maximized by a magnetic field guiding the electrons toward the electron detector, ${ }^{75}$ and the solid angle of the photon detection has been increased by a specifically designed aluminum mirror system. ${ }^{69}$

The time signals of the anode of the electron detector as well as of the photon detector's microchannel plates are measured as voltage drops in their respective high voltage supplies using capacitive coupling. Both signals are processed by fast amplifiers (RoentDek FAMP series) and constant fraction discriminators (RoentDek CFD7x) and then fed to a multichannel time-to-digital converter (TDC, RoentDek TDC8HP, $\Delta t_{\mathrm{RMS}}<35 \mathrm{ps}$ with a least significant bit length of $25 \mathrm{ps}$ ), which forward the arrival times with respect to the reference clock of the storage ring to the acquisition software. The reference clock is provided by BESSY II and is synchronized to the circulation of the bunch filling pattern. The temporal distance of two subsequent reference clock signals is $800 \mathrm{~ns}$ in the single bunch mode as well as the $\mathrm{MBH}$ mode and therefore does not provide any information about the bunch structure. The TDC measurement period is set to
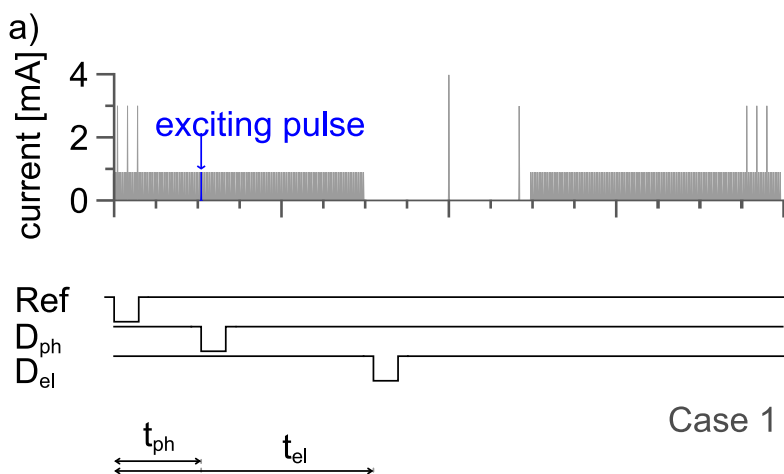

b)

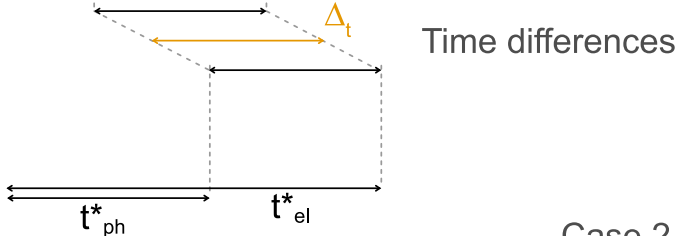

c)
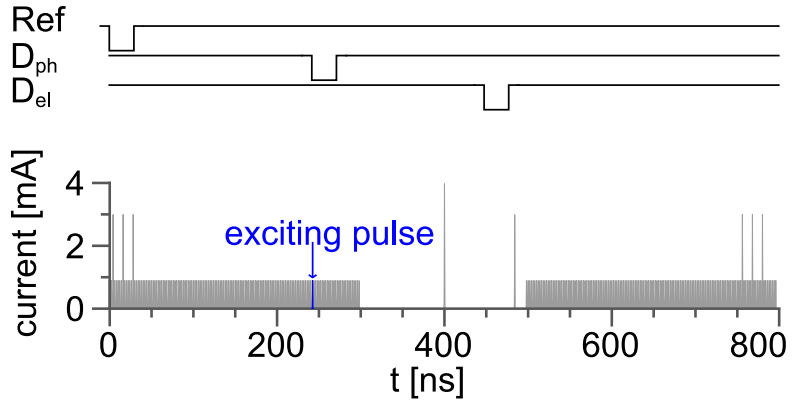

FIG. 1. Detection scheme for electron-photon coincidences using the multibunch hybrid mode of BESSY II, $, 6,63,72$ shown at the top of panel (a) and the bottom of panel (c). A simplified, schematic representation of the multibunch hybrid mode is shown as gray lines. The actual exciting bunches within the shown bunch periods are highlighted with blue markers. Each excitation event results in the detection of an electron and a photon. The respective signals are shown as pulses $D_{\text {ph }}$ (representing the photon detection time) and $\mathrm{D}_{\text {el }}$ (representing the electron time-of-flight) together with the BESSY bunch clock (Ref). The time-to-digital converter (TDC) is triggered by Ref and measures the times between the reference clock pulse and pulses of $D_{\text {ph }}$ and $D_{\text {el }}$ for each individual particle. (b) Particle-particle coincidences can be measured as a function of $\Delta_{\mathrm{t}}=t_{\mathrm{ph}}-t_{\mathrm{el}}$ to eliminate the floating offset between the reference pulse and time of excitation.

the time of two full bunch filling sequences, which corresponds to $1600 \mathrm{~ns}$. In the previous experiments using the single bunch mode, these settings allowed the quantification of accidental coincidences ${ }^{69}$ using a variation of background estimation approaches using multiple detection windows ${ }^{31,32,34}$ and digital coincidence counting, ${ }^{66-68}$ which is suitable for synchrotron radiation based experiments.

The results obtained by this method will be used for benchmarking the results obtained by the method discussed in the following. For the different data analysis methods, the signal processing and data acquisition are identical. The data acquisition 
scheme does not rely on specific coincidence detection cycles but measures all combinations of particles, i.e., single electrons, single photons, and any occurring combination of multiple electron and photon coincidences, using three input channels of the TDC (one channel for the reference clock and one channel per detector). Thus, the full information on the detection times of all individual particles relative to the start of their respective detection window is stored, enabling a flexible offline data treatment, as discussed above.

\section{DATA EVALUATION}

The measurement data consists of the electron time-of-flight $t_{\mathrm{el}}$ and the photon detection time $t_{\mathrm{ph}}$ of the respective particles relative to the reference clock signal (REF) provided by BESSY II (Fig. 1). For arbitrary excitation pulse sequences, the histogram of the number of recorded particles as a function of $t_{\mathrm{el}}$ or $t_{\mathrm{ph}}$ does, in general, not yield an unequivocal correlation between a detector event and an individual excitation pulse since the electron time-of-flights as well as the lifetime of the fluorescing states might be longer than the average time between subsequent excitation pulses. Instead, the data obtained are a convolution of the single-pulse time-of-flight spectrum and the excitation pulse sequence.

However, the histogram of the number of coincidences as a function of the relative detector arrival time

$$
\Delta_{\mathrm{t}}=t_{\mathrm{ph}}-t_{\mathrm{el}}
$$

can be used since true coincidences can be described by the convolution of the temporal distributions for the detection of the contributing individual particles after single-pulse excitation within this $\Delta_{\mathrm{t}}$-representation. For the electron-photon coincidences, it is the convolution of the temporal distribution of the photon signal and the electron signal. Figure 1 illustrates this for the case that all temporal distributions, i.e., the reference time and both types of detected particles, can be described by a delta distribution. If there are no further effects widening the temporal distributions of the detected particles, the relative detector arrival times of the coincident particles will be independent of the time difference between the reference clock and the actual excitation event.

In a more realistic, yet simplified example, we now consider the temporal distributions in the case of a single open channel for the electron emission preparing an excited ionic state. This state can only de-excite to one well-defined lower state by fluorescence. Hence, the photon distribution after a single-excitation pulse is approximated by the product of an exponential decay defined by the lifetime $\tau$ and a step function [Fig. 2(a), purple solid line], and the electron distribution can be approximated by a Gaussian caused by the apparatus function of the magnetic-bottle-type time-of-flight spectrometer [Fig. 2(a), green dashed-dotted line]. In this case, the distribution of relative detector arrival times can be described by the convolution of these two distributions, ${ }^{41}$

$$
\mathrm{P}^{\text {True }}\left(\Delta_{\mathrm{t}}\right)=\frac{\alpha}{2} \exp \left(\frac{\sigma^{2}}{2 \tau^{2}}-\frac{\Delta_{\mathrm{t}}-t_{0}}{\tau}\right) \cdot \operatorname{erfc}\left(\frac{\sigma}{\sqrt{2} \cdot \tau}-\frac{\Delta_{\mathrm{t}}-t_{0}}{\sqrt{2} \cdot \sigma}\right)+y_{0},
$$

where $\sigma$ is the standard deviation of the Gaussian, $\alpha$ is its area, $t_{0}$ is the center of the electron time-of-flight distribution in the $\Delta_{t}$ representation, $\tau$ is the lifetime of the radiative state, and $y_{0}$ is a possible a)

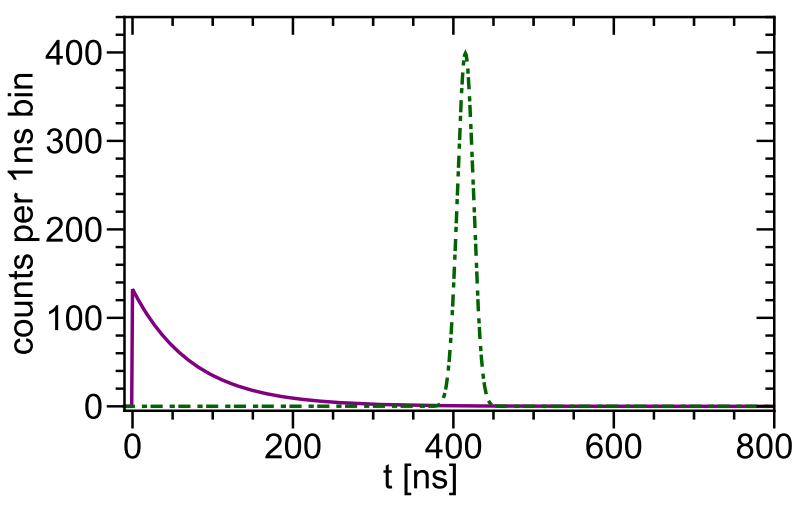

b)

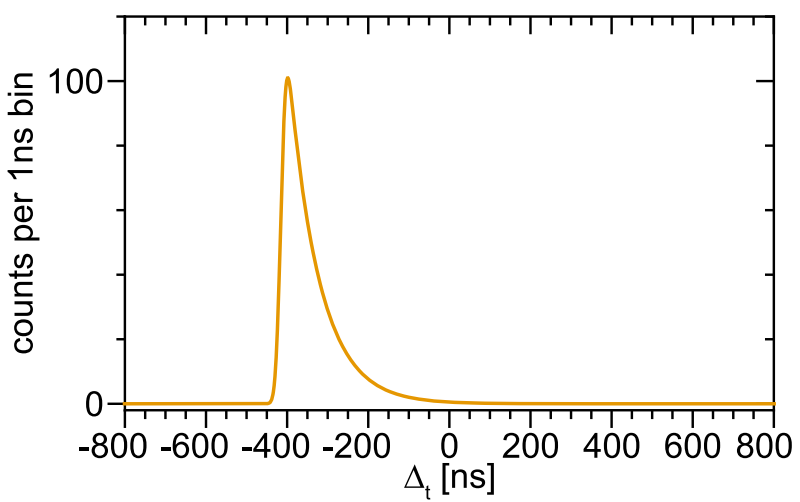

FIG. 2. (a) Exemplary simulated photon detection time distribution with a lifetime of the corresponding excited state of $\tau=75 \mathrm{~ns}$ (solid purple line) and an electron time-of-flight spectrum with a full width at half maximum FWHM $=23.5 \mathrm{~ns}$ (dashed-dotted green line) in the case of true electron-photon coincidences resulting from a single delta function excitation pulse at $t=0.0 \mathrm{~ns}$. The total number of electron-photon coincidences was set to $10^{4}$ events. (b) Resulting relative detector arrival time spectrum using Eq. (2) and the photon and electron distributions from panel (a).

offset to account for a possible constant background [Fig. 2(b)]. If electron-photon pairs from multiple decay channels are detected in coincidence, the total $\Delta_{\mathrm{t}}$-spectrum is comprised of an incoherent superposition of the contributing $\mathrm{P}^{\text {True }}\left(\Delta_{\mathrm{t}}\right)$ distributions. Analysis of these spectra might become challenging if the lifetimes $\tau$ of the involved states are too large compared to the temporal spacing between the time-of-flight of different electron channels due to strongly overlapping features.

It may happen that an electron and a photon are accidentally created in two independent target-projectile interactions within the acquisition window, either at two different target systems by two photons of the same excitation pulse, or by two separate pulses of the arbitrary fill pattern. This is also possible under single-excitation pulse conditions (e.g., using the single bunch filling mode of the storage ring). Accidental coincidences for single bunch excitation conditions have been removed from the coincidence spectra in the past using statistical reasoning and comparison of the spectra of two consecutive excitation pulses. The corresponding analysis procedure 
has been explained in detail in Ref. 69 and will be used here for benchmarking.

For arbitrary excitation patterns, however, this method cannot be used. For such patterns, accidental coincidences appear as a background below the distribution described by Eq. (2) and they can be accounted for by the following considerations. Accidental coincidences are caused by the photon probability distribution $P_{\mathrm{ph}}^{\mathrm{acc}}\left(t_{\mathrm{ph}}\right)$ and the electron probability distribution $P_{\mathrm{el}}^{\mathrm{acc}}\left(t_{\mathrm{el}}\right)$ generating the distribution of accidental coincidences $P^{\text {acc }}\left(\Delta_{\mathrm{t}}\right)$. However, no physical interdependence between these distributions exists, and therefore, all possible relative arrival times for any chosen measurement time window are possible and only determined by these probability distributions. Therefore, the distribution $P^{\text {acc }}\left(\Delta_{\mathrm{t}} ; t_{\mathrm{el}}^{\text {fixed }}\right)$ of accidental coincidences for an arbitrarily chosen but fixed electron detection time $t_{\mathrm{el}}^{\text {fixed }}$ can be written as

$$
P^{\mathrm{acc}}\left(\Delta_{\mathrm{t}} ; t_{\mathrm{el}}^{\mathrm{fixed}}\right)=P_{\mathrm{ph}}^{\mathrm{acc}}\left(t_{\mathrm{el}}^{\text {fixed }}+\Delta_{\mathrm{t}}\right) \cdot P_{\mathrm{el}}^{\mathrm{acc}}\left(t_{\mathrm{el}}^{\text {fixed }}\right) .
$$

Now, we compute the integral over all possible electron arrival times $t_{\mathrm{el}}$. The distribution of accidental events to be measured at $\Delta_{\mathrm{t}}$ can, therefore, be described by

$$
P^{\mathrm{acc}}\left(\Delta_{\mathrm{t}}\right)=\int_{t_{\mathrm{el}}} P_{\mathrm{ph}}^{\mathrm{acc}}\left(t_{\mathrm{el}}+\Delta_{\mathrm{t}}\right) \cdot P_{\mathrm{el}}^{\mathrm{acc}}\left(t_{\mathrm{el}}\right) \mathrm{d} t_{\mathrm{el}},
$$

which matches the definition of the cross correlation of the two independent, real-valued probability distributions $P_{\mathrm{ph}}^{\mathrm{acc}}\left(t_{\mathrm{ph}}\right)$ and $P_{\mathrm{el}}^{\mathrm{acc}}\left(t_{\mathrm{el}}\right)$ and agrees with the findings in Refs. 59 and 7. One can show that they can be identified by their respective non-coincident photon and electron distributions, i.e., with the non-coincident photon and electron spectra detected in the measurement window. Thus, the estimate of the background of accidental coincidences can be expressed by

$$
P^{\mathrm{acc}}\left(\Delta_{\mathrm{t}}\right)=\int_{t_{\mathrm{el}}} P_{\mathrm{ph}}^{\text {noncoinc }}\left(t_{\mathrm{el}}+\Delta_{\mathrm{t}}\right) \cdot P_{\mathrm{el}}^{\text {noncoinc }}\left(t_{\mathrm{el}}\right) \mathrm{d} t_{\mathrm{el}} \cdot
$$

The number of accidental coincidences for each $\Delta_{\mathrm{t}}$ can then be computed by multiplication of $P^{\text {acc }}\left(\Delta_{\mathrm{t}}\right)$ with the total number of accidental coincidences. Since this number is unknown, we treat it as a scaling factor for a given exciting-photon energy and target system, which is found by a least-square optimization. This optimization is done for a subset of the data, which consist of accidental coincidence events only. For this purpose, the settings of the electron spectrometer are chosen such that the longest possible electron time-of-flight does not exceed the time period of the excitation pattern $(800 \mathrm{~ns})$, but the TDC acquisition time is set to twice this value $(1600 \mathrm{~ns})$. Therefore, a time difference $\Delta_{\mathrm{t}}$ with $\Delta_{\mathrm{t}}<-800 \mathrm{~ns}$ is only possible for accidental electron-photon coincidence events with the electron and the photon originating from two independent interactions.

\section{RESULTS AND DISCUSSION}

The validity of the presented evaluation method for arbitrary excitation pulse sequences (AEPS) has been benchmarked by its application to a reference dataset recorded during a previous single bunch operation for a prototypical process after $2 p$ photoionization of Ar clusters. We recently showed how to use a combination of electron-photon and electron-electron coincidence spectroscopy to separate atomic and cluster Auger electron emission using the method for single-excitation pulses (SEP). ${ }^{78}$ Local Auger decay and subsequent charge redistribution within the clusters via radiative charge transfer (RCT) ${ }^{79,80}$ result in electron-photon coincidences. Figure 3(a) shows the electron time-of-flight spectrum (green dashed-dotted line) and the detection times of the measured photons (purple solid line) of all electron-photon coincidences for two subsequent excitation pulses of BESSY II operating in the single bunch mode.

The different features in the electron spectrum can be interpreted as Ar 2p photoelectrons and Ar Auger electrons, which are labeled in Fig. 3(c). For a detailed discussion of the underlying processes leading to the coincident emission of electrons and photons, see Ref. 78.

We now applied the present evaluation scheme to this dataset and obtained the corresponding $\Delta_{\mathrm{t}}$ spectrum [Fig. 3(b), orange solid line, obtained using Eq. (1)] as well as the background of accidental coincidences [Fig. 3(b), black dashed line, obtained using Eq. (5)]. Apparently, true coincidences appear, as expected, in a time difference of $800 \mathrm{~ns}$, namely between -800 and $0 \mathrm{~ns}$. In the regions $\Delta_{\mathrm{t}}<-800 \mathrm{~ns}$ and $\Delta_{\mathrm{t}}>0 \mathrm{~ns}$, the measured coincidences and the estimated accidental coincidence background cancel out for both regions, when scaled to the data in the region $\Delta_{\mathrm{t}}<-800 \mathrm{~ns}$. The resulting true electron-photon coincidence spectrum is shown as an orange line in Fig. 3(c). It is compared to the same dataset, evaluated by the SEP method described in Ref. 69 (green dotted line). One should note that the sign of the SEP dataset time axis was switched, and the spectrum was shifted by $+20 \mathrm{~ns}$ for better comparability.

This shift on the time axis between the SEP-method and the AEPS-method results from the sum of two terms. The first term stems from the time difference between the reference clock and the excitation pulse and is, therefore, eliminated by the $\Delta_{\mathrm{t}^{-}}$ representation of the coincidence data. The second term is caused by different processing times of the electron and photon signals resulting, e.g., from different cable lengths of the used electronics. This yields a total offset of the time difference spectrum for a given experimental setup, but can be accounted for during the conversion of the time-of-flight representation to the kinetic energy representation.

The results of the SEP analysis method are well reproduced by the AEPS method except for the described shift of the total spectrum. Although the AEPS method is based on the convolution of the electron time-of-flight spectrum and the exponential decay due to the radiative lifetime according to Eq. (2), the spectra analyzed by the SEP and AEPS method already agree without the convolution of the true electron and true photon signal. This is because the radiative lifetime $\tau$ of the observed process is about $5 \mathrm{~ns}$ (obtained from an exponential fit to the time dependence of the photon signal) and, therefore, short compared to the temporal width of the features observed in the electron spectrum. Thus, if $\tau$ is short compared to the width of the features in the electron spectrum, our approach directly yields the electron time-of-flight spectrum.

The capabilities of the AEPS method for data analysis have been tested with experiments performed in the $\mathrm{MBH}$ operation mode of BESSY II, recording electron-photon coincidences after photoionization and subsequent radiative decay of $\mathrm{CO}_{2}$ upon interaction with $22.0 \mathrm{eV}$ photons. Ionization by photons with an energy above 
a)

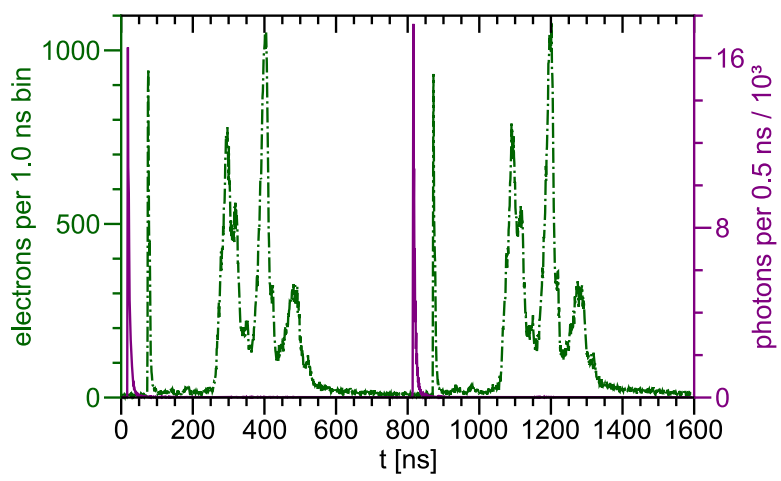

b)

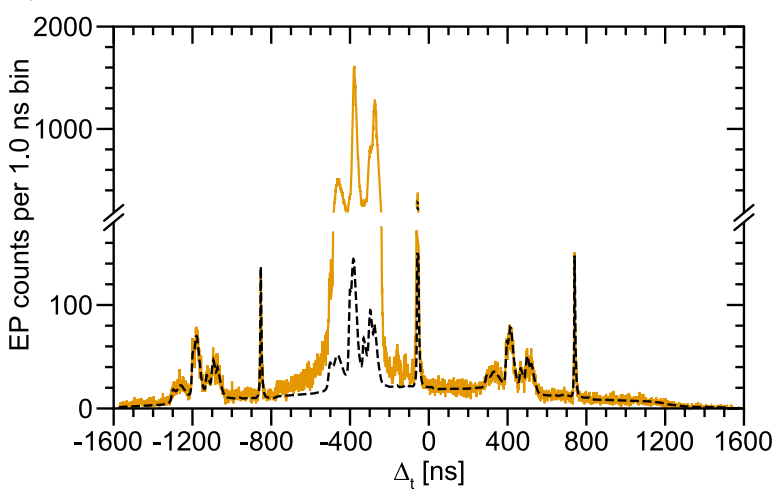

c)

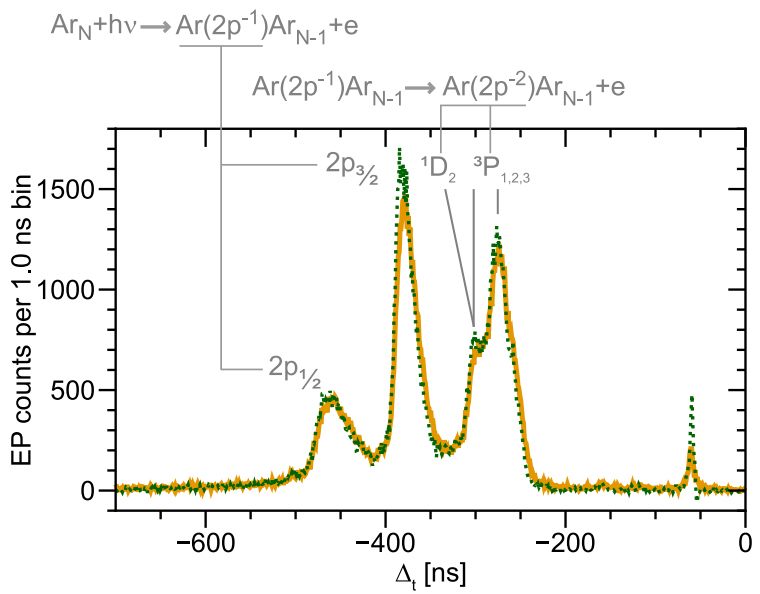

FIG. 3. Comparison of the two different evaluation schemes using data taken at BESSY II in the single bunch operation mode. ${ }^{78}$ (a) Recorded time-of-flight electron spectra of all electron-photon coincidences (true plus accidental) after $2 p$ photoionization of Ar clusters using an exciting-photon energy of $449 \mathrm{eV}$ and a retarding potential of $-190 \mathrm{~V}$ (green dashed-dotted line). The corresponding photon detection times are shown by the purple solid line. (b) $\Delta_{t}$-representation of all electron-photon coincidences (orange line) and the estimated background using the non-coincident data (black dashed line). Two different scales are used on the broken count axis to illustrate the full dataset. (c) Comparison of the SEP scheme (green dotted line) and AEPS scheme (orange solid line) of the true coincident electron spectra.
$17.6 \mathrm{eV}$ may populate excited electronic $\mathrm{CO}_{2}^{+} \mathrm{B}$ states. ${ }^{81}$ The subsequent $\mathrm{B}-\mathrm{X}$ transitions result in the emission of photons within the sensitivity range of the used photon detector and yield true electron-photon coincidences.

The evaluation of the coincident electron spectrum [Fig. 4(a), green dashed-dotted line] and photon detection times [Fig. 4(a), purple solid line] with respect to the reference clock does not yield meaningful information due to the arbitrary offset between the reference clock and the individual excitation events. The convolution of a single-excitation spectrum and the BESSY II MBH excitation pattern can be guessed. On the other hand, the $\Delta_{\mathrm{t}}$-representation of the electron-photon coincidences shows a clear structure on the top of a background [Fig. 4(b), orange solid line]. Additionally, the computed background using the non-coincident electron and photon data is shown as a black dashed line. This background matches with the data in the regions where only accidental coincidences are expected $\left(\Delta_{\mathrm{t}}<-800 \mathrm{~ns}\right.$ and $\left.\Delta_{\mathrm{t}}>800 \mathrm{~ns}\right)$, when scaled to the data in the region $\Delta_{\mathrm{t}}<-800 \mathrm{~ns}$. This is also confirmed by the difference of the coincidence data in its $\Delta_{\mathrm{t}}$-representation and the computed background [Fig. 4(c), orange solid line]. No artifacts from poor background estimation are observed and the subtraction yields a single structure on the top of some weak noise. The asymmetry of the structure already suggests that the radiative lifetime is not negligible compared to the width of the apparatus broadening of the electron spectrum.

We validated our evaluation procedure by determining the radiative lifetime $\tau$ as well as the electron time-of-flight (in the $\Delta_{\mathrm{t}}$ representation) by fitting Eq. (2) to the data [Fig. 4(c), black dotted line]. The obtained lifetime $\tau=(149.5 \pm 0.5)$ ns agrees with the range of reported lifetimes of the $\mathrm{CO}_{2}^{+} \mathrm{B}$ state based on coincidence measurements from 118 to $150 \mathrm{~ns} .{ }^{82,83}$ In the next step, the obtained coincidence signal was smoothed using a weighted moving average approach [Fig. 5(a), dotted line]. The relative weights stem from a normalized Gaussian distribution with $\sigma_{\text {smooth }}=2 \mathrm{~ns}$. While a direct deconvolution of the retrieved signal using inverse filtering ${ }^{84}$ yields only noise, the identical deconvolution method of the smoothed signal yields the electron spectrum [solid line in Fig. 5(a)]. This indicates high sensitivity of the used deconvolution procedure to the signal-to-noise ratio of the input data. The chosen $\sigma_{\text {smooth }}$ represents a trade-off between the signal-to-noise ratio of the deconvolved signal and a (in this case, negligible) influence on the resulting signal width. Subsequently, the deconvolved signal was converted to the electron kinetic energy representation [Fig. 5(b), solid line]. Here, the time-of-flight axis was calibrated to kinetic energies using electron-photon coincidence data resulting from photoionization and subsequent RCT in NeKr clusters. ${ }^{73}$ The center of this distribution is determined using a least-square optimization of a Gaussian [Fig. 5(b), dotted line] and results in an electron kinetic energy of $E_{\mathrm{El}, \text { kin }}=(3.84 \pm 0.06) \mathrm{eV}$, which corresponds to a binding energy of $E_{\mathrm{El} \text {, bind }}=(18.16 \pm 0.07) \mathrm{eV}$ for the exciting-photon energy of $h v=(22.01 \pm 0.01) \mathrm{eV}$. Here, the uncertainty of the kinetic energy stems from the statistical uncertainty of the least square optimization. The retrieved binding energy $E_{\mathrm{El} \text {, bind }}=(18.16 \pm 0.07) \mathrm{eV}$ shows good agreement with the already published data of $E_{\mathrm{El}, \text { bind }}^{(\mathrm{Lit})}$ $=18.1 \mathrm{eV} \cdot{ }^{81-83}$ While, in the present case, the radiative lifetime was obtained by the fitting procedure of the convoluted signal, for short lifetimes, it may be determined directly using the dark gap in the hybrid fill pattern. We estimate an upper boundary of this radiative 
a)

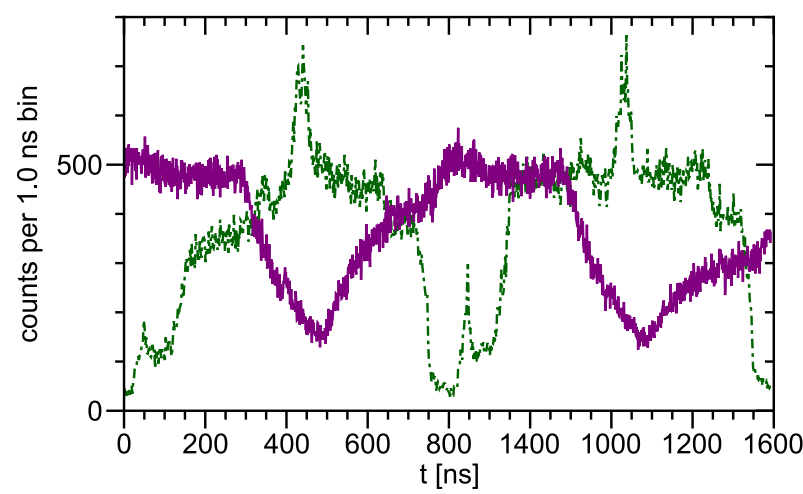

b)

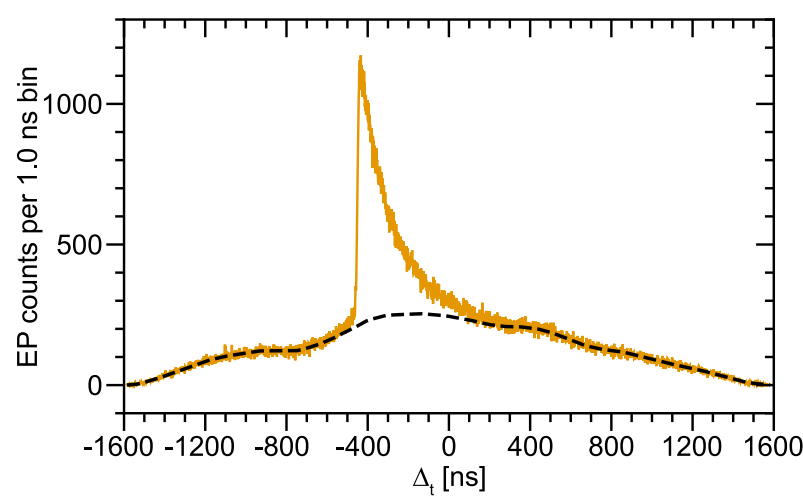

c)

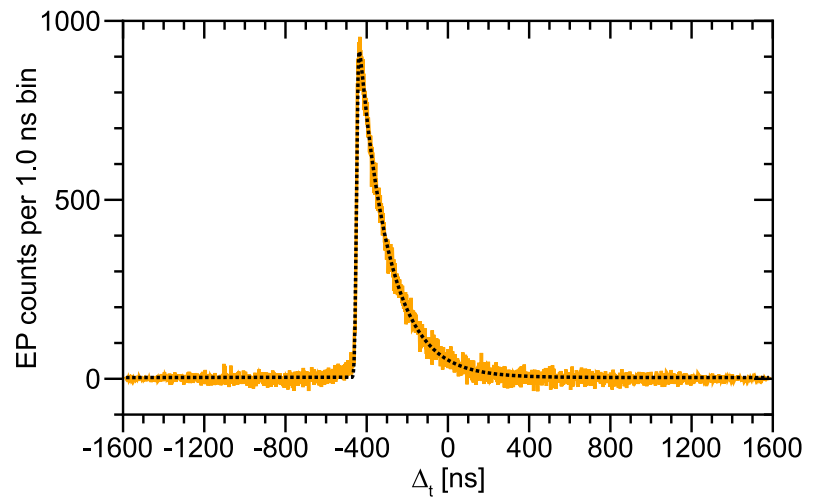

FIG. 4. (a) Recorded electron spectra of all electron-photon coincidences (true plus accidental) after photoionization of $\mathrm{CO}_{2}$ using an exciting-photon energy of $22.0 \mathrm{eV}$ and an acceleration potential of $+4 \mathrm{~V}$ (green dashed-dotted line) and the measured times of the corresponding emitted photons (purple solid line) using the multibunch hybrid mode of BESSY II. (b) $\Delta_{t}$-representation of the electron-photon coincidences (orange solid line) and estimated background using the non-coincident data (black dashed line). (c) Signal after background subtraction (orange solid line) and fit of the signal using Eq. (2), which is used to retrieve the radiative lifetime and the center of the distribution (black dotted line).

lifetime determination of $\tau_{\max } \approx 40$ ns for the 200 ns dark gap in the hybrid mode of BESSY II. For longer lifetimes, there will be a nonnegligible pile up from the multibunch part of the fill pattern. In the case of more complex spectra, which comprise multiple structures a)

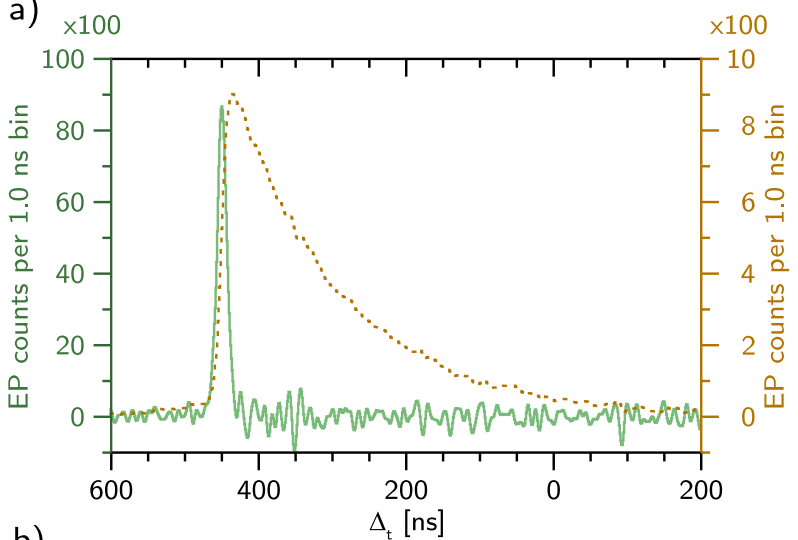

b)

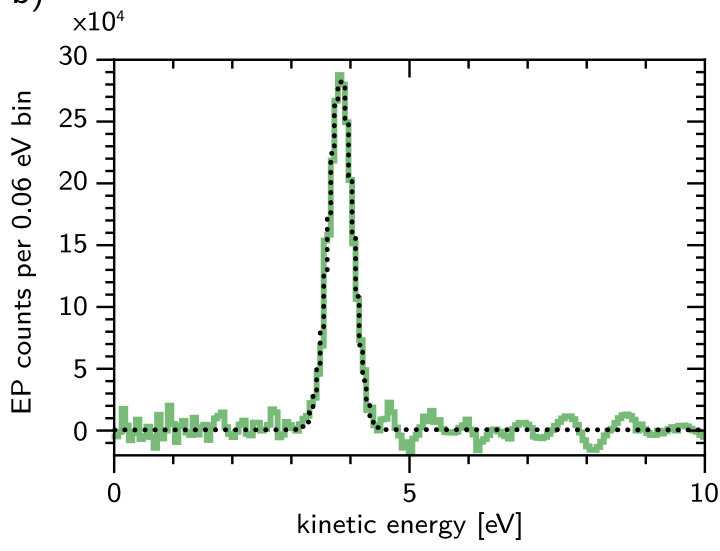

FIG. 5. (a) Moving average of the electron-photon coincidence data corresponding to Fig. 4(c) (dotted line and righthand y axis) and the deconvolved signal (solid line and lefthand $y$ axis) using the determined lifetime of the radiative states $\tau=(149.5 \pm 0.5) \mathrm{ns}$. (b) Kinetic energy spectrum of the deconvolved signal (solid line) and a Gaussian approximation to this signal (dotted line).

in the electron time-of-flight spectra and/or multiple radiative lifetimes, the deconvolution approach might become inapplicable. Here, measurements in the MBH mode might still be feasible if combined with appropriate advanced data evaluation methods, which inverse the data treatment, e.g., algorithms optimizing the estimated electron time-of-flight spectra and radiative lifetimes, and compare their convolution with the results from the $\mathrm{MBH}$ measurements or machine learning methods. This might be especially suited for processes for which some observables can be estimated by a priori calculations. If the electron time-of-flight is not a well-defined quantity (e.g., in the case of single-photon double ionization), the analysis may be adjusted to transfer the data to a better quantity (electron kinetic energies) prior to the fitting procedure.

\section{CONCLUSIONS}

A method for data analysis of electron-electron coincidence experiments upon excitation by arbitrary excitation pulse sequences (AEPS method) ${ }^{58-61}$ and neutron fission experiments ${ }^{7}$ has been transferred to photon-electron coincidence experiments. The 
method solely relies on detection times of the particles of interest and is independent of a common time reference. It has been applied to coincident electron-photon detection after photoionization of $\mathrm{CO}_{2}$ into excited B-states of $\mathrm{CO}_{2}^{+}$by an excitation pulse sequence delivered by the MBH mode of BESSY II. We showed that the background of accidental coincidences can be modeled using the non-coincident electron and photon time spectra even for temporal distances of subsequent excitation pulses as short as $2 \mathrm{~ns}$, confirming the validity of our approach for basically arbitrary excitation patterns. Prior to its application to the $\mathrm{CO}_{2}$-data, the AEPS evaluation procedure was benchmarked and validated by comparing its results to a previously published dataset analyzed in a standard evaluation procedure, which relies on well separated single-excitation pulses (SEP method). The results of both evaluation procedures agree within the experimental uncertainties. As the AEPS-method can be used for arbitrary excitation pulse sequences, it is more versatile compared to the SEP-method and enables faster data acquisition in coincidence experiments. If the radiative lifetime of the photon emission is short compared to the width of the features in the electron spectrum, our approach directly yields the coincident-electron time-of-flight spectrum as demonstrated by the example of coincident detection of Auger electrons and RCT photons from Ar clusters. In practice, this will be valid in many cases since the typical lifetime of fluorescence emission is in the order of hundreds of picosecond to few nanoseconds. Alternatively, a fitting and deconvolution procedure yields information on both the radiative lifetime and the electron time-offlight spectrum. The latter method requires either an isolated feature allowing for an adequate fitting as shown in the present work or knowledge about the radiative lifetime as the input parameter for the deconvolution. The showcase example of the $\mathrm{CO}_{2}$ photoionization into the $\mathrm{CO}_{2}^{+} \mathrm{B}$ satellite state and subsequent photon emission was investigated using the complete MBH mode of BESSY II. The obtained radiative lifetime $\tau=(149.5 \pm 0.5) \mathrm{ns}$ and the electron binding energy $E_{\mathrm{El} \text {, bind }}=(18.16 \pm 0.07) \mathrm{eV}$ are in good agreement with literature values. ${ }^{81-83}$ The presented approach is applicable to arbitrary excitation patterns and may stimulate efforts to include photon detection to other coincidence experiments, increasing both physical information obtained in an experiment and versatility of coincidence spectrometers. For more complex physical processes, the deconvolution of the measured signal might be replaced by the convolution of simulated signals in combination with optimization algorithms.

\section{ACKNOWLEDGMENTS}

We thank HZB for beamtime allocation and the BESSY II staff for assistance. This work was funded by the Deutsche Forschungsgemeinschaft (DFG) (Project No. 328961117 - SFB 1319 ELCH and Research Unit FOR 1789). The authors acknowledge the financial support from the Federal Ministry of Education and Research of Germany (BMBF) in the framework of Project No. 05K19RK2.

\section{DATA AVAILABILITY}

The data that support the findings of this study are available from the corresponding author upon reasonable request.

\section{REFERENCES}

${ }^{1}$ W. Bothe and H. Geiger, Z. Phys. 26, 44 (1924).

${ }^{2}$ W. Bothe and M. Born, Nobel Lectures, Physics 1942-1962 (Elsevier Publishing Company, Amsterdam, 1964).

${ }^{3}$ J. V. Dunworth, Rev. Sci. Instrum. 11, 167 (1940).

${ }^{4}$ R. B. Galloway, Phys. Educ. 5, 25 (1970).

${ }^{5}$ S. M. Judge, D. Arnold, B. Chauvenet, R. Collé, P. de Felice, E. García-Toraño, and U. Wätjen, Appl. Radiat. Isot. 87, 27 (2014).

${ }^{6}$ M. Demarteau, R. Lipton, H. Nicholson, and I. Shipsey, Rev. Mod. Phys. 88, 045007 (2016).

${ }^{7}$ J. M. O’Donnell, Nucl. Instrum. Methods Phys. Res., Sect. A 805, 87 (2016).

${ }^{8}$ K. Strauch, Rev. Sci. Instrum. 24, 283 (1953).

${ }^{9}$ R. Mankel, Rep. Prog. Phys. 67, 553 (2004).

${ }^{10}$ H. de Kerret, T. Abrahão et al., Nat. Phys. 16, 558 (2020).

${ }^{11}$ M. E. Phelps, E. J. Hoffman, N. A. Mullani, and M. M. Ter-Pogossian, J. Nucl. Med. 16, 210 (1975).

${ }^{12}$ M. M. Ter-Pogossian, M. E. Phelps, E. J. Hoffman, and N. A. Mullani, Radiology 114, 89 (1975).

${ }^{13}$ L. Bonolis, Am. J. Phys. 79, 1133 (2011).

${ }^{14}$ S. S. Kimura, K. Murase, P. Mészáros, and K. Kiuchi, Astrophys. J. 848, L4 (2017).

${ }^{15}$ M. Fiorentino, P. L. Voss, J. E. Sharping, and P. Kumar, IEEE Photonics Technol. Lett. 14, 983 (2002).

${ }^{16}$ J. Leach, B. Jack, J. Romero, A. K. Jha, A. M. Yao, S. Franke-Arnold, D. G. Ireland, R. W. Boyd, S. M. Barnett, and M. J. Padgett, Science 329, 662 (2010).

${ }^{17}$ J. H. D. Eland, Int. J. Mass Spectrom. 8, 143 (1972).

${ }^{18}$ R. E. Imhof and F. H. Read, Chem. Phys. Lett. 3, 652 (1969).

${ }^{19}$ J. H. D. Eland, M. Devoret, and S. Leach, Chem. Phys. Lett. 43, 97 (1976).

${ }^{20}$ R. D. DuBois, Nucl. Instrum. Methods Phys. Res., Sect. B 10-11, 120 (1985).

${ }^{21}$ J. L. Robins, Prog. Surf. Sci. 48, 167 (1995).

${ }^{22}$ P. Lablanquie, M. Lavollee, J. H. D. Eland, F. Penent, and R. I. Hall, Meas. Sci, Technol. 6, 939 (1995).

${ }^{23}$ R. Dörner, V. Mergel, O. Jagutzki, L. Spielberger, J. Ullrich, R. Moshammer, and H. Schmidt-Böcking, Phys. Rep. 330, 95 (2000).

${ }^{24}$ R. E. Continetti, Annu. Rev. Phys. Chem. 52, 165 (2001).

${ }^{25}$ J. Ullrich, R. Moshammer, A. Dorn, R. Dörner, L. P. H. Schmidt, and H. Schmidt-Böcking, Rep. Prog. Phys. 66, 1463 (2003).

${ }^{{ }^{26}} \mathrm{~T}$. Arion and U. Hergenhahn, J. Electron Spectrosc. Relat. Phenom. 200, 222 (2015).

${ }^{27}$ P. Lablanquie, M. A. Khalal, L. Andric, J. Palaudoux, F. Penent, J.-M. Bizau, D. Cubaynes, K. Jänkälä, Y. Hikosaka, K. Ito, K. Bučar, and M. Żitnik, J. Electron Spectrosc. Relat. Phenom. 220, 125 (2017).

${ }^{28}$ R. M. Littauer, Rev. Sci. Instrum. 21, 750 (1950).

${ }^{29}$ I. Powis and P. Downie, Rev. Sci. Instrum. 69, 3142 (1998).

${ }^{30}$ C. Giffin, A. Kaufman, and W. Broecker, J. Geophys. Res. 68, 1749, https://doi.org/10.1029/jz068i006p01749 (1963).

${ }^{31}$ K. Parodi, P. Crespo, H. Eickhoff, T. Haberer, J. Pawelke, D. Schardt, and W. Enghardt, Nucl. Instrum. Methods Phys. Res., Sect. A 545, 446 (2005).

${ }^{32}$ J.-Y. Yu, Z. Wang, and S.-M. Chen, Chin. Phys. C 39, 056201 (2015).

${ }^{33}$ R. Britton, J. L. Burnett, A. V. Davies, and P. H. Regan, Nucl. Instrum. Methods Phys. Res., Sect. A 769, 20 (2015).

${ }^{34}$ J. F. Oliver and M. Rafecas, PLoS One 11, e0162096 (2016).

${ }^{35}$ C. Eckart and F. R. Shonka, Phys. Rev. 53, 752 (1938).

${ }^{36}$ J. Bryant, Int. J. Appl. Radiat. Isot. 14, 143 (1963).

${ }^{37}$ E. M. Friedländer, Nucl. Instrum. Methods 31, 293 (1964).

${ }^{38}$ D. Smith, Nucl. Instrum. Methods 152, 505 (1978).

${ }^{39}$ H. Kossmann, Meas. Sci. Technol. 4, 16 (1993).

${ }^{40}$ M.-S. Lu and T. Teichmann, Nucl. Instrum. Methods Phys. Res., Sect. A 327, 544 (1993).

${ }^{41}$ N. H. Gale, Nucl. Phys. 38, 252 (1962).

${ }^{42}$ R. E. Imhof and F. H. Read, J. Phys. B: At., Mol. Phys. 4, 450 (1971).

${ }^{43}$ G. C. King, F. H. Read, and R. E. Imhof, J. Phys. B: At., Mol. Phys. 8, 665 (1975). 
${ }^{44}$ E. W. Schlag, R. Frey, B. Gotchev, W. B. Peatman, and H. Pollak, Chem. Phys, Lett. 51, 406 (1977).

${ }^{45}$ N. Bose, J. Phys. B: At., Mol. Phys. 11, L309 (1978).

${ }^{46}$ K. A. Mohamed and G. C. King, J. Phys. B: At., Mol. Phys. 12, 2809 (1979).

${ }^{47}$ G. Dujardin, S. Leach, and G. Taieb, Chem. Phys. 46, 407 (1980).

${ }^{48}$ T. Field and J. H. D. Eland, Chem. Phys. Lett. 197, 542 (1992).

${ }^{49}$ M. Meyer, J. Lacoursière, M. Simon, and P. Morin, Rev. Sci. Instrum. 66, 1554 (1995).

${ }^{50}$ M. Kitajima, M. Ukai, S. Machida, K. Kameta, A. Ehresmann, N. Kouchi, Y. Hatano, T. Hayaishi, E. Shigemasa, and K. Ito, J. Phys. B: At., Mol. Opt. Phys. 29, 1711 (1996).

${ }^{51}$ M. Doğan and A. Crowe, ARI - Int. J. Phys. Eng. Sci. 51, 34 (1998).

${ }^{52}$ M. Ries, J. Feikes, T. Goetsch, P. Goslawski, J. Li, M. Ruprecht, A. Schälicke, and G. Wüstefeld, in Proceedings of the 6th International Particle Accelerator Conference (IPAC'15), Richmond, VA, USA, May 3-8, 2015, International Particle Accelerator Conference No. 6 (JACoW, Geneva, Switzerland, 2015), pp. 138-140.

${ }^{53}$ R. Müller, T. Birke, F. Falkenstern, K. Holldack, A. Jankowiak, M. Ries, and A. Schälicke, in Proceedings of International Particle Accelerator Conference (IPAC'17), International Particle Accelerator Conference No. 8 (JACoW, Geneva, Switzerland, 2017), pp. 2574-2577.

${ }^{54}$ K. Holldack, R. Ovsyannikov, P. Kuske, R. Müller, A. Schälicke, M. Scheer, M. Gorgoi, D. Kühn, T. Leitner, S. Svensson, N. Mårtensson, and A. Föhlisch, Nat. Commun. 5, 4010 (2014).

${ }^{55}$ Y. Hikosaka, Rev. Sci. Instrum. 90, 053105 (2019).

${ }^{56}$ D. F. Förster, B. Lindenau, M. Leyendecker, F. Janssen, C. Winkler, F. O. Schumann, J. Kirschner, K. Holldack, and A. Föhlisch, Opt. Lett. 40, 2265 (2015).

${ }^{57}$ S. Plogmaker, P. Linusson, J. H. D. Eland, N. Baker, E. M. J. Johansson, H. Rensmo, R. Feifel, and H. Siegbahn, Rev. Sci. Instrum. 83, 013115 (2012).

${ }^{58}$ E. Jensen, R. A. Bartynski, S. L. Hulbert, and E. D. Johnson, Rev. Sci. Instrum. 63, 3013 (1992).

${ }^{59}$ P. Calicchia, S. Lagomarsino, F. Scarinci, C. Martinelli, and V. Formoso, Rev. Sci. Instrum. 70, 3529 (1999).

${ }^{60}$ P. Bolognesi, M. Coreno, G. Alberti, R. Richter, R. Sankari, and L. Avaldi, J. Electron Spectrosc. Relat. Phenom. 141, 105 (2004)

${ }^{61}$ V. Ulrich, S. Barth, T. Lischke, S. Joshi, T. Arion, M. Mucke, M. Förstel, A. M. Bradshaw, and U. Hergenhahn, J. Electron Spectrosc. Relat. Phenom. 183, 70 (2011).

${ }^{62}$ K. Holldack, M. v. Hartrott, F. Hoeft, O. Neitzke, E. Bauch, and M. Wahl, Proc. SPIE 6771, 677118 (2007).

${ }^{63}$ P. Goslawski, J. Feikes, K. Holldack, A. Jankowiak, R. Ovsyannikov, M. Ries, M. Ruprecht, A. Schälicke, and G. Wüstefeld, in Proceedings of International Particle Accelerator Conference (IPAC'16), International Particle Accelerator Conference No. 7 (JACoW, Geneva, Switzerland, 2016), pp. 3427-3430.
${ }^{64}$ O. Kugeler, S. Marburger, and U. Hergenhahn, Rev. Sci. Instrum. 74, 3955 (2003).

${ }^{65}$ T. Gießel, D. Bröcker, P. Schmidt, and W. Widdra, Rev. Sci. Instrum. 74, 4620 (2003).

${ }^{66}$ S. M. Buckman and D. Ius, Nucl. Instrum. Methods Phys. Res., Sect. A 369, 368 (1996).

${ }^{67}$ M. Havelka, P. Auerbach, and J. Sochorová, Appl. Radiat. Isot. 56, 265 (2002).

${ }^{68}$ G. Prümper, Y. Tamenori, A. de Fanis, U. Hergenhahn, M. Kitajima, M. Hoshino, H. Tanaka, and K. Ueda, J. Phys. B: At., Mol. Opt. Phys. 38, 1 (2005).

${ }^{69}$ A. Hans, C. Ozga, P. Schmidt, G. Hartmann, A. Nehls, P. Wenzel, C. Richter, C. Lant, X. Holzapfel, J. H. Viehmann, U. Hergenhahn, A. Ehresmann, and A. Knie, Rev. Sci. Instrum. 90, 093104 (2019).

${ }^{70}$ G. Reichardt, J. Bahrdt, J.-S. Schmidt, W. Gudat, A. Ehresmann, R. MüllerAlbrecht, H. Molter, H. Schmoranzer, M. Martins, N. Schwentner, and S. Sasaki, Nucl. Instrum. Methods Phys. Res., Sect. A 467-468, 462 (2001).

${ }^{71}$ P. Baumgärtel and I. Packe, and Helmholtz-Zentrum Berlin für Materialien und Energie, J. Large-Scale Res. Facil. 2, A53 (2016).

${ }^{72}$ A. Lüdeke, M. Bieler, R. H. A. Farias, S. Krecic, R. Müller, M. Pont, and M. Takao, Phys. Rev. Accel. Beams 19, 082802 (2016).

${ }^{73}$ A. Hans, T. Miteva, X. Holzapfel, C. Ozga, P. Schmidt, H. Otto, G. Hartmann, C. Richter, N. Sisourat, A. Ehresmann, K. Gokhberg, U. Hergenhahn, and A. Knie, Phys. Rev. Lett. 123, 213001 (2019).

${ }^{74}$ J. S. Miller, E. D. Poliakoff, T. F. Miller, A. P. P. Natalense, and R. R. Lucchese, J. Chem. Phys, 114, 4496 (2001)

${ }^{75}$ M. Mucke, M. Förstel, T. Lischke, T. Arion, A. M. Bradshaw, and U. Hergenhahn, Rev. Sci. Instrum. 83, 063106 (2012).

${ }^{76}$ O. Jagutzki, A. Cerezo, A. Czasch, R. Dorner, M. Hattas, M. Huang, V. Mergel, U. Spillmann, K. Ullmann-Pfleger, T. Weber, H. Schmidt-Bocking, and G. D. W. Smith, IEEE Trans. Nucl. Sci. 49, 2477 (2002).

${ }^{77}$ A. Hans, P. Schmidt, C. Ozga, G. Hartmann, X. Holzapfel, A. Ehresmann, and A. Knie, Materials 11, 869 (2018).

${ }^{78}$ A. Hans, C. Küstner-Wetekam, P. Schmidt, C. Ozga, X. Holzapfel, H. Otto, C. Zindel, C. Richter, L. S. Cederbaum, A. Ehresmann, U. Hergenhahn, N. V. Kryzhevoi, and A. Knie, Phys. Rev. Res. 2, 012022(R) (2020).

${ }^{79}$ N. Saito, Y. Morishita, I. H. Suzuki, S. D. Stoychev, A. I. Kuleff, L. S. Cederbaum, X.-J. Liu, H. Fukuzawa, G. Prümper, and K. Ueda, Chem. Phys. Lett. 441, 16 (2007).

${ }^{80}$ S. D. Stoychev, A. I. Kuleff, F. Tarantelli, and L. S. Cederbaum, J. Chem. Phys. 128, 014307 (2008).

${ }^{81}$ Q. Tian, J. Yang, Y. Shi, X. Shan, and X. Chen, J. Chem. Phys. 136, 094306 (2012).

${ }^{82}$ C. Herran, F. Arqueros, and J. Campos, J. Mol. Spectrosc. 97, 244 (1983).

${ }^{83}$ M. A. Johnson, R. N. Zare, J. Rostas, and S. Leach, J. Chem. Phys. 80, 2407 (1984).

${ }^{84}$ P. Virtanen, R. Gommers, T. E. Oliphant et al., Nat. Methods 17, 261 (2020). 